\title{
Studying a light sensor with light: multiphoton imaging in the retina
}

Thomas Euler ${ }^{1,2 *}$, Katrin Franke ${ }^{1,3}, \&$ Tom Baden ${ }^{1,4}$

${ }^{1}$ Institute for Ophthalmic Research, University of Tübingen, Germany

${ }^{2}$ Werner Reichardt Centre for Integrative Neuroscience (CIN), University of Tübingen, Germany

${ }^{3}$ Bernstein Centre for Computational Neuroscience Tübingen, University of Tübingen, Germany

${ }^{4}$ School of Life Sciences, University of Sussex, Brighton, UK

* Corresponding author

\section{i. Summary/Abstract}

Two-photon imaging of light stimulus-evoked neuronal activity has been used to study all neuron classes in the vertebrate retina, from the photoreceptors to the retinal ganglion cells. Clearly, the ability to study retinal circuits down to the level of single synapses or zoomed out at the level of complete populations of neurons, has been a major asset in our understanding of this beautiful circuit. In this chapter, we discuss the possibilities and pitfalls of using an all-optical approach in this highly light-sensitive part of the brain.

\section{ii. Key Words}

Vertebrate retina, mouse, zebrafish, two-photon microscopy, biosensor, activity probes, visual stimulus-evoked activity, laser-evoked retinal activity 


\section{Introduction}

Unlike most other neuronal tissues, the retina directly responds to light. Accordingly, using any form of light microscopy to study its function inevitably leads to superimposition of neuronal activity driven by the "intended" visual stimulus with activity driven by the optical imaging system itself. Since single-photon (1P) excitation of fluorescence, as used in traditional wide-field charge-coupled device (CCD) camera systems or for confocal microscopy, usually uses excitation light within the visual spectrum, this conflict is near insurmountable. Typically, the excitation light of these imaging systems would saturate or even permanently blind the photoreceptors of the retina, thus leaving little room for modulation of their activity using additional sources of light. Two-photon (2P) microscopy [1] dramatically ameliorates - though never eliminates - this problem by using an infrared laser outside the visual spectrum for fluorescence excitation. This way, direct activation of retinal photoreceptor cells by the imaging system becomes much less of a problem [2-4]. As a result, $2 \mathrm{P}$ microscopy as a tool for studying neuronal function revolutionised retinal research over the past decades. At last, researchers could draw on the power of optical imaging to complement their physiological toolkit. Here, following a brief recap of retinal structure and function, we will highlight the advantages and remaining pitfalls of using $2 \mathrm{P}$ imaging to study this highly light-sensitive tissue with optical recording techniques, and how the light-induced side effects of the actual measurement can be estimated and overcome.

\subsection{Probing retinal function with optical methods}

The retina comprises five principal classes of neurons that are organised into three nuclear and two synaptic layers (Fig. 1a). In the outer plexiform layer (OPL), photoreceptors connect to the dendrites of horizontal cells and bipolar cells. The latter in turn carry the signal to the inner plexiform layer (IPL) where they contact amacrine cells as well as the dendrites of retinal ganglion cells (RGCs), the eye's projection neurons to the brain. Most computational "power" of the retinal network derives from its two synaptic layers as well as from final signal integration by the RGCs. Accordingly, most studies of 
retinal function have focussed on these sites. As for neuronal networks in other parts of the brain, the ability of $2 \mathrm{P}$ microscopy to resolve neuronal activity down to the level of single synapses here really comes to shine.

With a few notable exceptions, most studies looking at vertebrate retinal function using $2 \mathrm{P}$ microscopy have focussed on the retinae of mice, rabbits and larval zebrafish. Initially dominated by use of synthetic fluorescent probes introduced by single-cell injections (Fig. 1b), nowadays genetically-encoded indicators of neuronal activity ("biosensors") are used in genetically accessible model systems such as mouse and zebrafish (Fig. 1c-f). Such biosensors include, for instance, the GCaMP family of calcium $\left(\mathrm{Ca}^{2+}\right)$ probes $[5,6]$ or, more recently, iGluSnFR, a sensor for extracellular glutamate [7-9]. For example, Dreosti et al. [10] expressed SyGCaMP2 under the RibeyeA promotor to drive expression of this $\mathrm{Ca}^{2+}$ biosensor in bipolar cells of the larval zebrafish retina. This was followed by a series of studies using this approach and its subsequent iterations using other forms of GCaMP or SypHy to study zebrafish inner retinal processing at great depth [11-17]. In parallel, $\mathrm{Ca}^{2+}$ imaging using biosensors also found its way into the mouse retina (Fig. 1c,e) $[5,18]$. This enabled investigating more complex synaptic interactions with multiple partners $[19,20]$, such as those at the core of the intensely studied "direction-selective" (DS) circuit in the retina [21]. Even intracellular chloride $\left(\mathrm{Cl}^{-}\right)$levels - indicative of inhibitory synaptic inputs mediated by $\mathrm{\gamma}$-aminobutyric acid (GABA) or glycine receptors - can be monitored in this way. For example, Duebel et al. [22] used $\mathrm{Cl}^{-}$imaging to reveal in certain bipolar cell types a standing $\mathrm{Cl}^{-}$gradient, which enables these cells to process GABAergic inputs differentially at the dendrites and the axon terminals. More recently, the ascent of glutamate imaging further expanded the available toolset for studying retinal function (Fig. 1d,f; [7, 23]). All major excitatory neurons of the retina use glutamate as their neurotransmitter. Accordingly, the ability to directly monitor their synaptic output using glutamate imaging - rather than "just" the presynaptic $\mathrm{Ca}^{2+}$ signals that drive release - turned out to be yet another game changer. For example, Franke et al. [24] surveyed light-driven glutamate release across the entire mouse IPL to provide a functional fingerprint of each bipolar cell type and in turn used this to study global rules of 
inhibition in the inner retina [24]. In the meanwhile, others used this approach to study receptor types underlying transient and sustained Off bipolar cell responses [25] and the role of excitation in direction selectivity [26].
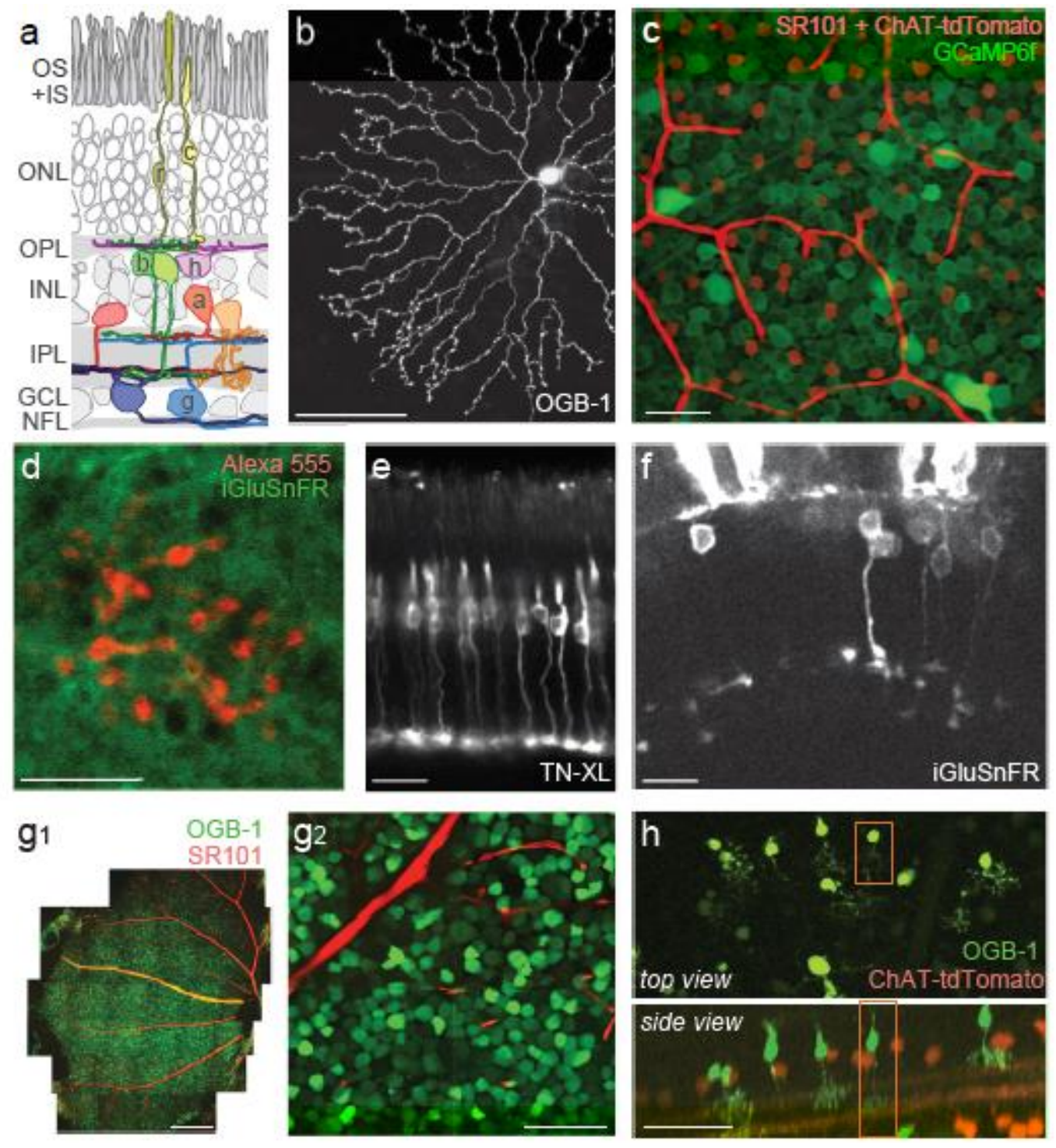

Fig. 1. Retina and retinal labeling

Imaging of genetically encoded and targeted probes aside, retinal research also made major headway using single-cell or population labelling of retinal neurons using synthetic fluorescent Ca ${ }^{2+}$ indicators (Fig. 1b,g,h). For example, in an early study, Euler et al. [27] imaged starburst amacrine cells in the rabbit retina following single-cell dye loading (Fig. 1b) to uncover a major mechanism in these cells that supports the extraction of motion information in the aforementioned DS circuit. 
Multiple studies followed suit using similar techniques, for example, most recently demonstrating species differences in these cells' dendritic wiring with the presynaptic partners [28]. Finally, given that the retina is a thin sheet of tissue, also bulk loading of synthetic indicators, in particular via electroporation (Fig. 1g,h), can be used to address questions that require complete labelling of neuronal populations, such as the synaptic terminals of photoreceptors in the OPL [29] or the cell bodies in the ganglion cell layer (GCL) [30-32]. Bulk loading with synthetic indicators may also be tweaked to result in sparse labelling useful for imaging individual neurons [33, 34], avoiding the need for technically challenging single-cell injections.

Taken together, $2 \mathrm{P}$ imaging has to date been used to study all major sites in the inner and outer retina, including cone pedicles [4, 29, 30, 35], horizontal cell dendrites [36], bipolar cell axon terminals $[20,26,34,37]$, amacrine cell circuits $[17,19,27,28,38-41]$ and ganglion cells $[20,30,32$, $34,42,43]$. Clearly, the ability to study retinal circuits down to the level of single synapses, or zoomed out at the level of complete populations of neurons, has been a major asset in our understanding of this beautiful circuit. However, as we shall see, we must continue to be vigilant about the remaining pitfalls of using an optical approach.

\subsection{Direct and indirect excitation of the retina in 2P microscopy}

Optical access to the retina from the photoreceptor side is limited, because here several layers of photoreceptor cell bodies form a light-scattering lenslet array (cf. Fig. 1a; [44]). Instead, the isolated and "flat-mounted" retina is typically accessed from the retinal ganglion cell side. However, as the retinal tissue itself is only around $150-200 \mu \mathrm{m}$ thick and transparent, the main challenge is not depth penetration - as it is in other parts of the brain - but rather light-sensitivity. Perhaps contrary to intuition, the infrared (IR) laser used in 2P microscopy is not at all invisible to the retina [2, 3].

The typical wavelength of the 2P excitation laser ranges between 850 and $1050 \mathrm{~nm}$, far beyond the peak of the spectral sensitivity curve of even the long-wavelength (L-) sensitive photopigments (Lopsins; peak sensitivity around $600 \mathrm{~nm}$, depending on the species [45]). However, all opsins have a 
long, ultra-low sensitivity tail that reaches far into the IR range [46]. Combined with the high laser power required for generating fluorescence signals, this direct (1P) excitation of opsins can be substantial (Fig. 2a). In addition, the 2P effect [47] can also excite opsins [2] (Fig. 2b) - just like the fluorescent probes. Due to the quadratic laser intensity dependence of 2P excitation (see Section 3.4) and the fact that scanning usually occurs in a plane several tens of micrometres away from the photoreceptors, 2P excitation of opsins appears highly unlikely. However, because of the extreme opsin concentration in the photoreceptor outer segments and the high gain of the phototransduction cascade, even sparse $2 \mathrm{P}$ opsin activation can contribute strongly to the laser-evoked response of the retina. Finally, the fluorescence that is meant to be generated within the tissue adds to this laser response: when scattered towards the photoreceptors, it hits the opsins right in the bulk of their sensitivity curve (Fig. 2c). This effect can cause rhythmic activity as the laser focus repetitively scans across a cell's receptive field. This effect is referred to as "indirect excitation" (of the photoreceptors) and further contributes to setting up a background activation of the retinal network.

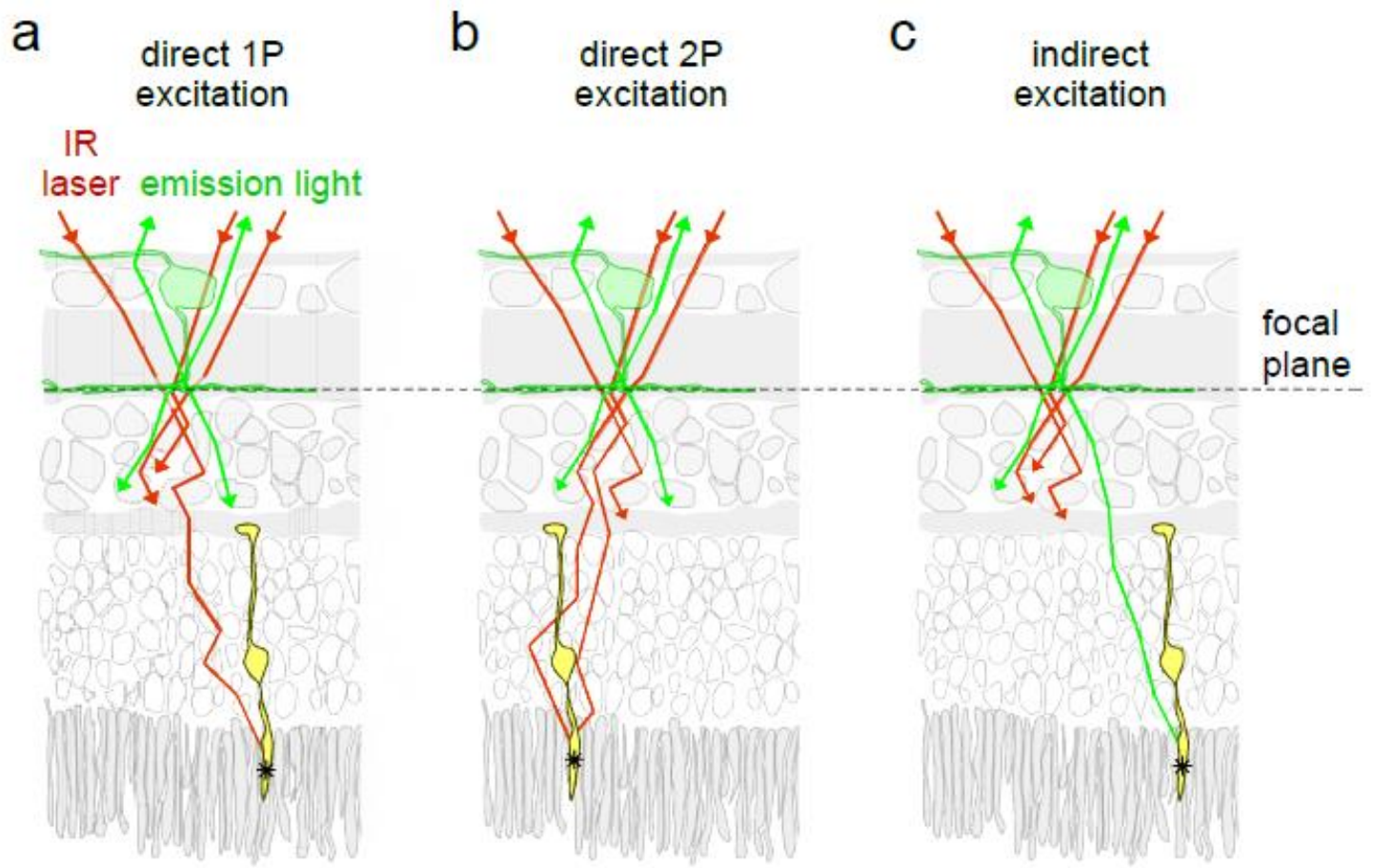

Fig. 2. Direct and indirect excitation of PRs 
Together, direct $1 \mathrm{P}$ and $2 \mathrm{P}$ excitation as well as indirect excitation of photoreceptors generate a "low-photopic" level of background activation in the retina [3]. This makes it difficult, if not impossible, to study retinal processing outside the photopic regime using $2 \mathrm{P}$ microscopy. How to estimate and minimise these effects is discussed in Section 3.4.

\subsection{Visual stimulation}

Beside the imaging system interfering with the photoreceptors and thereby triggering the retinal network ("laser effect", see Section 1.2), the possibility of "opposite" crosstalk presents a further complication: Any light introduced in addition to the excitation laser - for example, to provide a controlled visual stimulus to the retina - is potentially "seen" by the microscope's light detectors (some variant of photomultiplier tube, PMT). In other words, the visual stimulus can easily "swamp" the detection part of the 2P-system, thus making it impossible to image anything at all (or even permanently damage the PMTs). Two main approaches can be used to ameliorate this problem: (i) segregation of the spectrum into specific bands for fluorescence emission (from the probe) and light stimulation, for instance, using customised dichroic mirrors, or (ii) rapid temporal separation of image acquisition and visual stimulation. Ideally, both are used in conjunction [3].

Spectral separation is usually easy to achieve as visual stimulation does not need to cover the retina's full spectral sensitivity range. Depending on the species, two to four narrow wavelength bands are typically sufficient to differentially drive the spectrally different opsins (photoreceptors) and thus address basic questions in colour vision. In this way, conflicts between visual stimulation and imaging can be avoided, so long as two spectral windows for detection of the common fluorescent probes, which usually exhibit their fluorescent peaks around $530 \mathrm{~nm}$ ("green") and $630 \mathrm{~nm}$ ("red"), are left reserved for imaging. Example arrangements for mouse and zebrafish are given in Section 3.3.

However, due to the extreme light sensitivity of the microscope's PMTs and because filters never completely block light outside their pass-band(s), spectral segregation alone is usually not sufficient to satisfactorily ameliorate stimulation artefacts in the scans. Even if these are barely detectable, 
such stimulation artefacts hamper the reliable detection of the actual light stimulus-evoked activity. This problem can be addressed by segregating periods of image acquisition from those of light stimulation, for instance, by stimulating solely during the scan retrace (see Section 3.3 ). In addition, "gated" PMTs that, for instance, contain a shutter that can be driven with microsecond precision (see, e.g. the $\mathrm{H} 11526$ series by Hamamatsu), maybe become an option to prevent stimulation light from entering the actual detectors.

\subsection{Recording configurations}

Though principally transparent itself, the retina embedded in the pigmented eyeball remains difficult to optically access in vivo. As a result, most work on retinal physiology has been done in so-called "retinal explants" where the retina is removed in its entirety from the eyeball (see Section 3.1). In its "flattened" form, this in vitro configuration provides for straight-forward optical access of any retinal neuron and synaptic layer, and it simplifies spatially structured visual stimulation. Since the retinal network is largely feed-forward with most species receiving little or no centrifugal projections from the brain [48], this "whole-mount" configuration is considered to provide a reasonable approximation for most retinal functions.

An alternative in vitro configuration is the retinal slice - here, the retina is, for instance, mounted on a filter paper and sliced vertically into 200 to $300 \mu \mathrm{m}$-thick sections using a mechanical chopper [49]. Such slices provide direct electrophysiological access to all classes of retinal neurons and enable powerful combinations of single-cell 2P imaging and electrical recording (e.g. [38]). However, with techniques for targeting and visualising neurons deep in the tissue, whole-mounts are quickly catching up in this respect. In terms of imaging, slices have the additional advantage that layers close to the light-sensitive photoreceptor outer segments, such as the outer plexiform layer (OPL), become more accessible for optical recordings $[4,50,51]$ : with the axis of the excitation laser then running perpendicular to the retinal layering, direct $1 \mathrm{P}$ and $2 \mathrm{P}$ opsin excitation (see Sections $1.2,3.4$ ) is substantially reduced. The main downside of the slice is that crucial lateral connections in the retinal 
network are severed by the sectioning. Therefore, from a functional point of view, whole-mounts are preferable as here the long-range inhibitory interactions (i.e. polyaxonal amacrine cells [52] and horizontal cells [53]) remain intact.

In principle, in vivo imaging of the mammalian retina is also possible. This can be achieved using adaptive optics which permit optically corrected access of the imaging system through the cornea and lens and has been demonstrated, for example, in mice and macaques $[54,55]$, in the latter, though, not yet for 2P imaging of neuronal activity. However, this in vivo approach is technically challenging and therefore not yet widely used. In contrast, small, semi-transparent model species, such as the larval zebrafish, offer a simpler alternative for many research questions. Here, genetic (e.g. "crystal fish", [56]) or chemical removal of some of the eye's pigment [10] allow direct optical access to most of the retinal network in vivo. One remaining downside is that any removal of the eye's screening pigment around the eyeball also interferes with the spatial profile of light reaching different parts of the eye. Of course, this is also an issue in any form of retinal explant.

\section{Materials}

\subsection{Two-photon microscope}

In principle, any type of upright 2P microscope that allows visualising the selected fluorescent probe(s) at sufficient spatial and temporal resolution can be used. In our case, a through-theobjective (TTO) solution for the visual stimulator is employed [3]. For that, the scope must have the option for coupling in the stimulus into the main optical path. Visual stimulation from below the recording chamber - through-the-condenser (TTC) - is somewhat easier to set up but requires access to the sub-stage space and a condenser (or objective) lens of sufficient optical quality for projecting the stimulus onto the retina in the recording chamber (for details, see Section 2.2). Moreover, if the PMTs are positioned above the objective lens, as is often the case, sub-stage visual stimulation may run the risk of photon-swamping the detectors with the stimulus (see above). 
In our labs, we use MOM-type 2P microscopes, originally designed by W. Denk (MPI for Neurobiology, Martinsried, Germany) and now built and further developed by Sutter Instrument (Novato, CA, USA). We chose the MOM - the "movable objective microscope" - because it can be easily customised, e.g. by adding different types of visual stimulators (cf. Section 2.2). For a detailed description of the MOM design, see elsewhere [3]. In brief, our systems are equipped with a modelocked Ti:Sapphire laser (MaiTai-HP DeepSee, Newport Spectra-Physics, Darmstadt, Germany) tuneable to wavelengths roughly between 800 and $1030 \mathrm{~nm}$, a water immersion objective (e.g. W Plan-Apochromat 20x/1.0 DIC M27 VIS-IR, Zeiss, Oberkochen, Germany) and two fluorescence detection channels. The latter are optimised for detection of green and red fluorescent probes by using appropriate band-pass filters positioned in front of the PMTs (cf. Fig. 5a; for example, HQ 510/84 and HQ 630/60 dichroic filters, respectively; AHF Tübingen, Germany). For image acquisition, we use custom software ("ScanM" by M. Müller, Max Planck Gesellschaft, Martinsried, and T.E.) running under IGOR Pro 6.3 for Windows (Wavemetrics, Lake Oswego, OR, USA) on a PC equipped with fast multi-channel I/O cards (PCI-6110 or PCI-6115, and PCle-6363, National Instruments, München, Germany), which control scanners and image acquisition, and generate blanking and trigger signals (cf. Section 3.3). However, other software packages (e.g. ScanImage, Vidrio Technologies, Ashburn, VA, USA) can be used on the same hardware as well. In addition, Scanlmage is quite flexible and can be adapted to a range of imaging hardware and I/O cards.

\subsection{Visual stimulation}

The advantages of TTO stimulation (cf. Section 2.1 ) are (i) that the high quality of the objective lens used for imaging is also exploited for stimulus projection; (ii) that, assuming proper alignment, the stimulation centre is always registered to the centre of the imaging field (the stimulus is "carried around" with the imaging field when moving across the retinal surface); and (iii) that only a relatively small fraction of the stimulation light (the portion that is reflected from the tissue) re-enters the objective lens and needs to be prevented from reaching the PMTs, which in the case of the MOM are positioned above the objective. The main disadvantage of TTO is that the objective lens must provide 
sufficient spatial resolution (i.e. a high NA) while having a large field-of-view (FOV) and, thus, large stimulation area. Here, a 20x objective like the selected one (cf. Section 2.1) represents a good compromise for many research questions in the retina.

The TTO solution presented in this chapter uses a digital-mirror-device (DMD) based video projector that is coupled into the optical path of the microscope after the scan mirrors. Commercially available "consumer" devices can be employed, however, depending on the species studied and, thus, the wavelengths needed for effective opsin stimulation, the internal LEDs may need to be replaced by appropriate LED/filter combinations (for mouse and zebrafish solutions, see Section 3.3). For minimising cross-talk between light stimulator and PMTs (cf. Section 1.3), a modification of the LED control may be necessary to blank the stimulator light during the scanning (see Section 3.3). While such consumer devices are very affordable, they undergo fast revision cycles, such that only a year later it may be difficult to find the same model for which the LED modification was developed. Therefore, employing more expensive, but well-documented and controllable evaluation platforms with longer revision cycles, such as the LightCrafter ${ }^{\mathrm{TM}}$ (e.g. DLP LightCrafter 4500, Texas Instruments, Dallas, TX, USA), may be considered as an alternative. These devices are also available with customer-specified LEDs and appropriately optimised internal optics (e.g. EKB Technologies Ltd, BatYam, Israel) and even as a version with a port for a light-guide, which facilitates adding other light sources. The LightCrafter can be driven like a computer monitor via an HDMI link from any visual stimulation software (e.g. Psychtoolbox, http://psychtoolbox.org, [57]; or our own software, QDSpy, https://github.com/eulerlab/QDSpy, see Section 3.3).

For full-field stimuli, a simple, LED-based light stimulator in TTC configuration may be sufficient. Such a contraption can be easily built from a set of filters and LEDs driven by off-the-shelf electronics, like an Arduino Uno microcontroller board (https://www.arduino.cc/). It enables easy control of the LEDs' intensities via pulse-width modulation (PWM), precise timing of the stimulus time course, and simple synchronisation between image acquisition and stimulus presentation. For a possible design, see elsewhere $[58,59]$. 


\section{Methods}

\subsection{Tissue preparation}

The tissue preparation procedures for retinal imaging experiments are very similar to those used for standard physiological recordings in the retina, which is why we will not go into detail here. A stepby-step description of mouse retina dissection, including retinal slicing and whole-mount preparation, as well as a list of tools and materials, is available elsewhere (e.g. $[50,60])$. In the following, we will discuss a few guidelines regarding tissue preparation that are important for successfully imaging light-driven activity in the mouse retina. While most of these "rules" apply to any retinal experiment using light stimulation, some are critical for 2P imaging of the retina.

To maximise the amount of available light-sensitive visual pigment in the in vitro retina, animals should be dark-adapted for $\geq 1$ hour prior to dissection. In our experience, dark-adaptation also facilitates detaching the neural retina from pigment epithelium (PE), which is advantageous when isolating the retina from the eyecup. While helpful in the mouse, this step is essential in many nonmammalian preparations such as birds and fish where the outer segments of photoreceptors markedly move in and out of the pigment epithelium depending on the adaptation state [61]. To minimise visual pigment bleaching, we carry out all steps of the dissection procedure using illumination light at the long-wavelength edge of the species' visible spectrum: In mice, with their visual spectrum shifted to shorter wavelengths [62], very dim red light (e.g. 650 nm-LEDs with 100 $\mu \mathrm{W}$ power) still well-visible to humans, can be used. Minimising bleaching is less critical for preparations with the PE attached (e.g. eyecup), as in vertebrates, the visual pigment is mainly regenerated in the PE [63] and, therefore, the continuous supply with restored visual pigment is ensured also in vitro. While the dissection of the retina with attached PE is possible and routinely done in some species, including, e.g., Macaque monkeys [64], it is more challenging in rodents (for procedure, see [65]) and therefore rarely used. 
Depending on the research question, registering the orientation of the retina throughout the preparation procedure and the experiment may be critical. This is because in most, if not all species, the distribution of neurons across the retina is anatomically and/or functionally inhomogeneous (e.g. $[66,67])$. For example, in mice, short- and medium-wavelength sensitive photoreceptor types are unequally distributed across the retina $[59,68,69]$. In addition, recent studies demonstrate that different types of mouse RGCs show distinct distributions across the retina to differentially sample visual space [70-73]. One possibility to keep track of the retina's orientation during the dissection is to use an orienting mark (e.g. dorsal) on the eye whilst still in the animal and make a little cut in the eyecup (with the retina still in) at the corresponding location. Alternatively, the stereotypic pattern of retinal blood vessels may be used (for mice, see Fig. 2 in [74]). As a result, this allows monitoring the retinal position and orientation of the recording site(s), which can be crucial for data interpretation. 

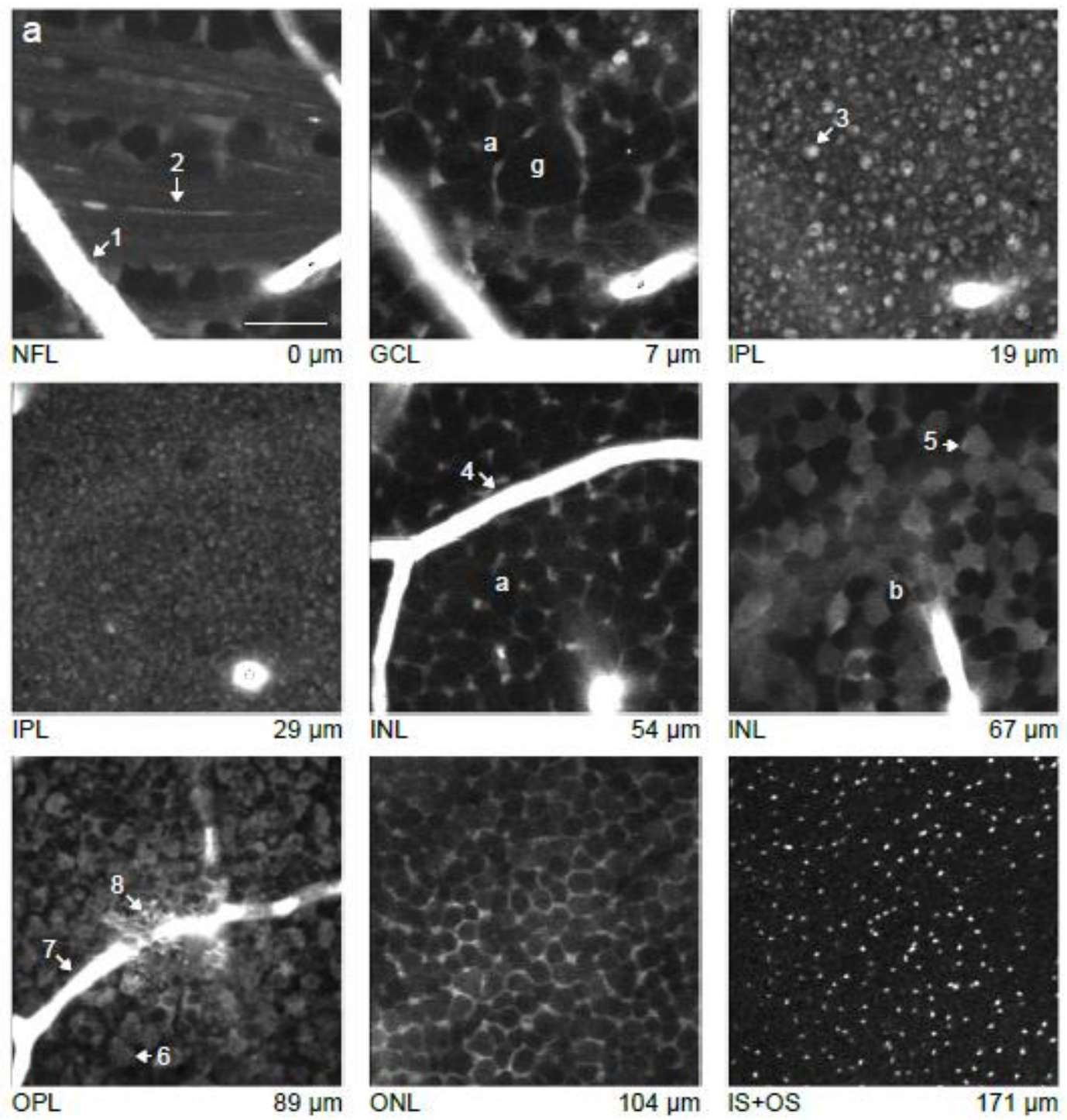

b

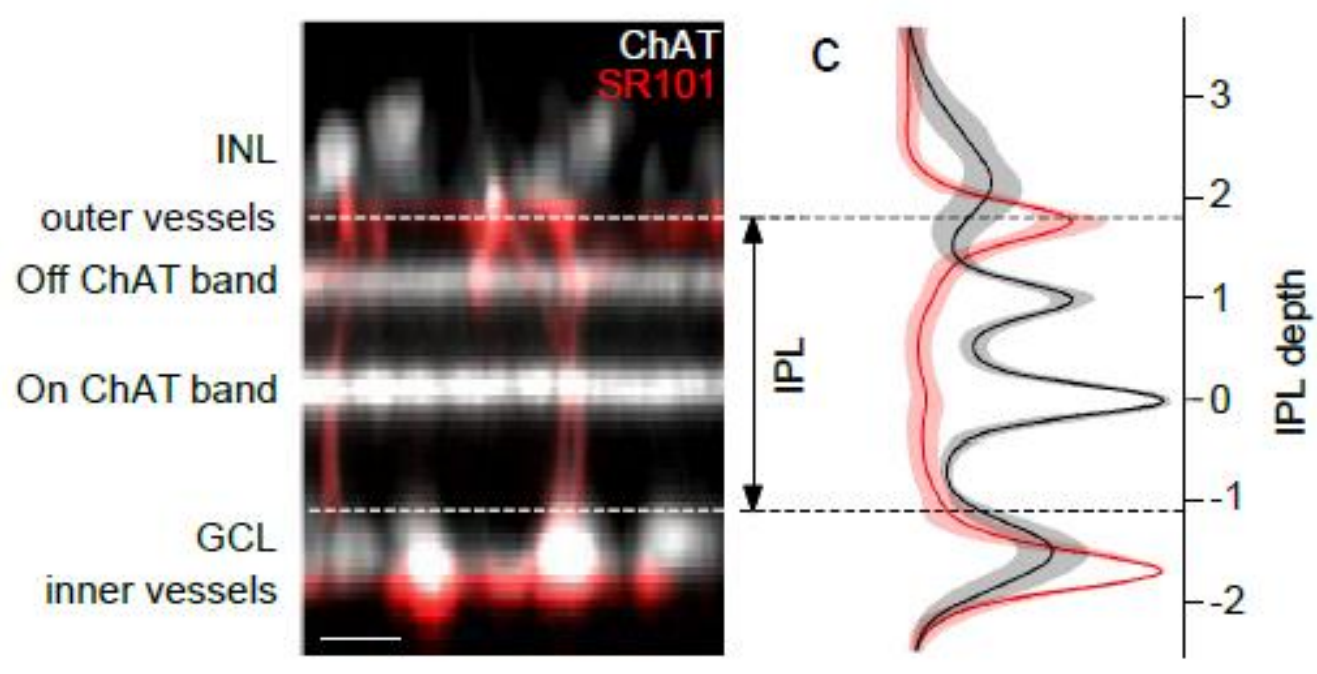

Fig. 3. Visualizing retinal structure 
As discussed in Section 1.4, retinal whole-mounts or slices can be chosen. While both preparations allow keeping track of the recording position (for preparing slices, see e.g. [36]), whole-mounts make it particularly easy to map positions in the explanted retina onto spherical coordinates, thereby reconstructing the recording site(s) in the intact, three-dimensional retina (e.g. using the "Retistruct" algorithm, [75]). This presents the researcher with the opportunity, to relate retinal coordinates, for example, to motion axes [76] or visual coordinates, thus providing insights into the functional role and potentially, behavioural relevance of the retinal cells and circuits studied.

When mounting the retina in the recording chamber, the stimulus direction needs to be considered. The TTO configuration - with the stimulus projected from above and the GCL up - allows "more intact" preparations, such as an eyecup or a PE-attached whole-mount. Here, the retina can be mounted on a non-transparent filter paper (e.g. nitrocellulose membrane, $0.8 \mu \mathrm{m}$ pore size, Millipore, Ireland) or on IR-transmissive ceramic discs ("Anodisc", \#13, $0.2 \mu \mathrm{m}$ pore size, GE Healthcare, USA). For the TTO configuration, however, the stimulated area is limited by the field of view of the objective (cf. Section 2.2). In contrast, when the stimulus is projected from below through a condenser, the retina needs to be detached from PE and sclera. In addition, the tissue needs to be mounted on filter paper (see above) in which a small window has been cut (to allow the stimulus from below reaching the photoreceptors). Alternatively, a "harp" (e.g. a "U"-shaped Pt-wire with nylon strings, self-made or from companies such as Warner Instruments, Hamden, CT, USA) can be used to keep the retina flat and steady in the recording chamber. Another option is using the transparent ceramic discs described above - however, one needs to consider that these discs scatter short-wavelength light, resulting in a blurred stimulus. Specifically, we found that $385 \mathrm{~nm}$-stimuli smaller than $50 \mu \mathrm{m}$ were blurred, whereas $576 \mathrm{~nm}$-stimuli of the same size were accurately projected on the retina.

If compatible with the other fluorescent (activity) probes used, $0.1 \mu \mathrm{M}$ of the red dye Sulforhodamine-101 (SR101, \#S359, Invitrogen) can be added to the perfusion. SR101 diffuses in the extracellular space and thereby visualises the retinal structure under the $2 \mathrm{P}$ microscope (Fig. 3a). It 
also enters damaged cells and brightly labels these $[3,77]$, thereby allowing to monitor tissue health during recordings. In addition, SR101 strongly stains retinal blood vessels (Fig. 3a), which can be used as a reliable stratification marker in the IPL (Fig. 3b,c; [24]) and as landmarks in the whole-mount, for example, to match recorded with subsequently immunolabeled cells [30]. Note that synaptically very active cells slowly take up SR101 by endocytosis (Fig. 3a); this has, for instance, been used to identify cone axon terminals in slices [36]. The level of SR101 staining usually allows distinguishing between this uptake-dependent labelling and labelling of damaged cells: The latter are stained quickly and become much brighter [77].

Finally, two more general points are in our experience critical for ensuring high quality recordings in the in vitro retina. First, recordings should be carried out at physiological temperatures $\left(\sim 37^{\circ} \mathrm{C}\right.$ for mouse, or typically $\sim 28^{\circ} \mathrm{C}$ for zebrafish - although as ectotherms, the fish retina is more temperature-resistant) to maximise photoreceptor light responses. Second, the perfusion should run with at least 3-4 $\mathrm{ml}$ per minute to keep the tissue healthy and responsive to light. In our experience, both artificial cerebral spinal fluid (ACSF) solution (for recipe, see for example [30]) and Ames' medium (e.g. A1420, Sigma-Aldrich / Merck, Darmstadt, Germany; [78]) work well for the isolated mouse retina.

\subsection{Labelling the retina with activity probes}

In recent years, the diversity of available indicators to measure different aspects of neuronal activity increased tremendously (e.g. [79]). This presents the opportunity to not only record neuronal activity per se but a specific biological process like neurotransmitter release (cf. Section 1.1). Here, we will briefly describe a selection of labelling techniques that were successfully employed to introduce activity probes into the retina and that enable recordings of light-driven responses across all retinal cell classes.

Genetically encoded fluorescent probes (biosensors), such as the GCaMP family [5, 6] or iGluSnFR [7] can be introduced into the tissue by a viral approach or stably expressed in transgenic animal lines. 
The great advantage of biosensors over synthetic dyes is the possibility to address neuronal subsets selectively, including single, genetically defined cell types. For example, we use mouse lines expressing the $\mathrm{Ca}^{2+}$ sensor TN-XL and GCaMP3 in cone photoreceptors and horizontal cells $[4,36]$, respectively, to image light-evoked $\mathrm{Ca}^{2+}$ changes in cone axon terminals (Fig. 4a) and horizontal cell dendrites (Fig. 4b) in the outer retina. While transgenic mouse lines have the advantage of stable expression suitable for longitudinal studies (e.g. [80]), their generation is very time consuming. Here, viral delivery of the biosensors, for instance, by transfection with adeno-associated virus (AAV) constitutes a more flexible alternative [81]. Viral vectors can be delivered to the retina via two routes [82]: They can be injected into the vitreous body of the eye ("intravitreal"), which predominantly targets inner retinal neurons, or into the subretinal space ("epiretinal"), targeting outer retinal neurons including photoreceptors.

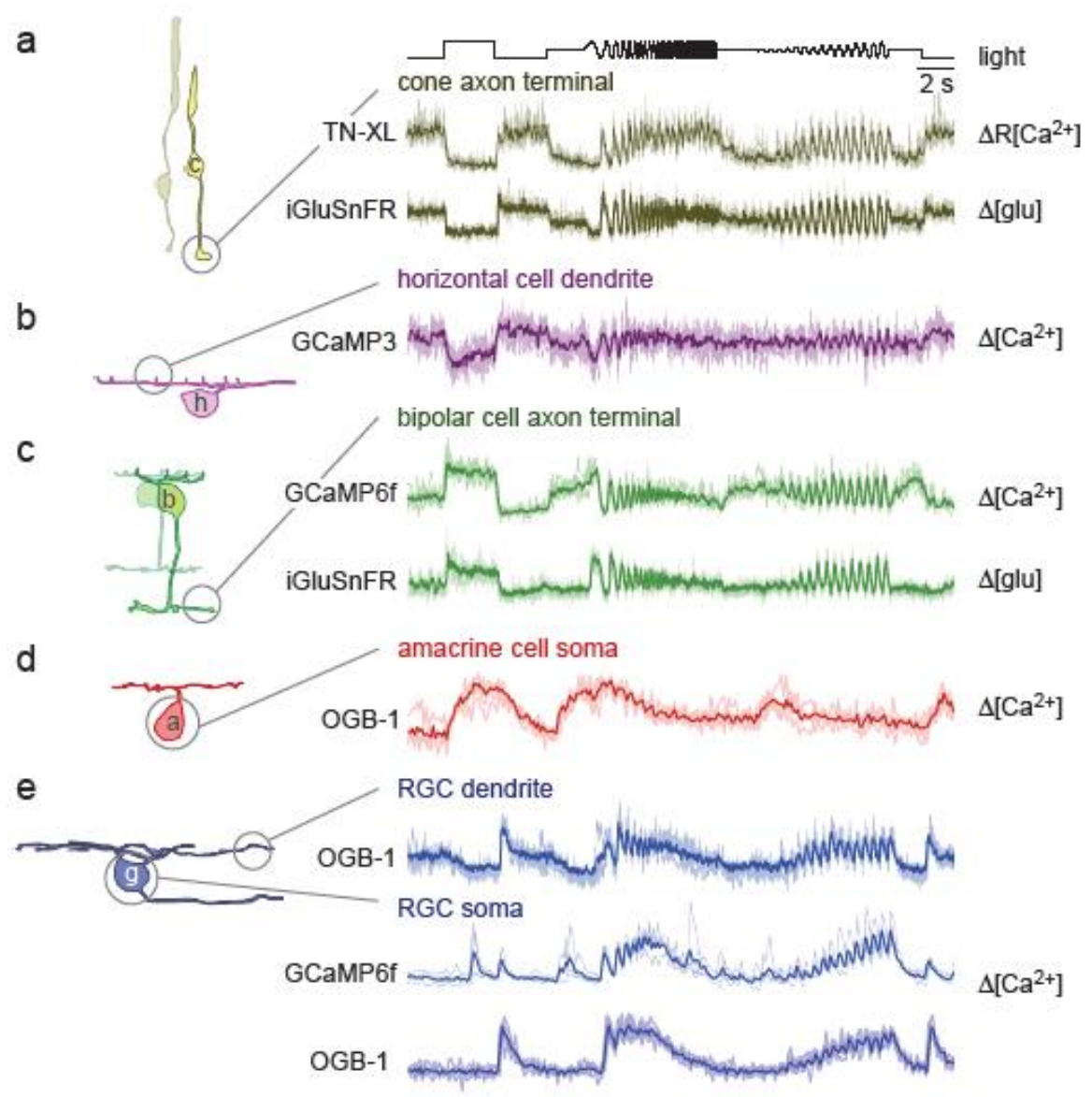

Fig. 4. Example responses 
We successfully injected AAVs purchased from Penn Vector Core (Philadelphia, PA, USA) encoding, e.g., the $\mathrm{Ca}^{2+}$ sensor GCaMP6f ([5], e.g. AAV9.hSyn.GCaMP6f), or the glutamate sensor iGluSnFR ([7], e.g. AAV2.hSyn.iGluSnFR) intravitreally using micromanipulators (M3301, WPI, Friedberg, Germany) and a Hamilton injection system (Hamilton Messtechnik $\mathrm{GmbH}$, Höchst, Germany); for a description of the procedure, see e.g. [24]. Red fluorescent versions of $\mathrm{Ca}^{2+}$ and glutamate biosensors, such as RGECO [83] and R-iGluSnFR1 (8), respectively, are also available. Depending on the combination of viral vector and mouse line used, the expression can be targeted to a subpopulation of cells or (more or less) ubiquitously expressed across cell types (see also below). For example, by injecting the "floxed" GCaMP6f virus into the Pcp2-cre line (JAX 006207, The Jackson Laboratory, Bar Harbor, USA), the $\mathrm{Ca}^{2+}$ sensor can be selectively expressed in a subset of bipolar cells, allowing, e.g., recording light-driven $\mathrm{Ca}^{2+}$ changes in isolated bipolar cell axon terminal systems (Fig. 4c). In contrast, ubiquitous expression of a $\mathrm{Ca}^{2+}$ indicator such as GCaMP6f allows recording somatic light responses across a population of RGC types in the scan field (Fig. 4e). Similarly, the glutamatergic output of all bipolar cell types and photoreceptors can be systematically recorded by expressing the iGluSnFR ubiquitously across the retina (Fig. 4a,e). In general, the retinal transduction efficiency strongly depends on the serotype of AAV used: While some serotypes like AAV2 or AAV2.7m8 efficiently pass the inner limiting membrane [84], other serotypes, such as AAV9 or AAV1, require a punctuation of the inner limiting membrane with the injection needle to access the retina.

While synthetic dyes lack the cell type selectivity and stable expression of biosensors (see above), their easy delivery to the tissue (cf. Section 1.1) as well as the variety of available dyes (reviewed in [85]) make them a good alternative for imaging neuronal activity. Membrane impermeable forms of synthetic dyes can be introduced into single cells or population of cells by electroporation (e.g. [31]). For example, injecting individual RGCs with the $\mathrm{Ca}^{2+}$ sensor Oregon-Green BAPTA-1 (OGB-1) labels single dendritic trees, which allows to record light-driven responses in different dendritic segments of the same cell (Fig. 4e). In contrast, bulk-electroporation of the retina using established $\mathrm{Ca}^{2+}$ dyes like 
OGB-1 and Rhod-2, or more recent ones, such as Cal-520 [86], allows imaging somatic responses of complete populations of GCL cells [30], including RGCs (Fig. 4e) and displaced amacrine cells (Fig. 4d).

\subsection{Light stimulation}

As a visual stimulator for mice, we employ a customized DMD projector (DLP LightCrafter 4500, see Section 2.2) (Fig. 5a), equipped with a UV $\left(\lambda_{\text {peak }}=390 \mathrm{~nm}\right)$ and a green $\left(\lambda_{\text {peak }}=\sim 520 \mathrm{~nm}\right)$ LED, whose emission spectra are shaped by a dual-band dichroic filter (DM2, F59-003, AHF, Tübingen, Germany). The projector is connected via HDMI to a Microsoft Windows PC running the visual stimulation software QDSpy (https://github.com/eulerlab/QDSpy) written in Python. It allows presentation of arbitrary visual stimuli and movies controlled by user-written Python scripts and supports generation of trigger signals (for off-line analysis of synchronisation of stimulus presentation and acquired imaging data) via a dedicated I/O card (e.g. PCI-DIO24, Measurement Computing, BietigheimBissingen, Germany) or any Arduino microcontroller. The visual stimulus is coupled into the main optical path of the $2 \mathrm{P}$ microscope and projected into the tissue via the objective lens (TTO arrangement). To this end, a cold mirror (CM in Fig. 5a) is placed just in front of the scan mirrors, where it transmits the excitation laser and reflects the light stimulus towards the scan lens (for details on the optical arrangement, see [3]). 

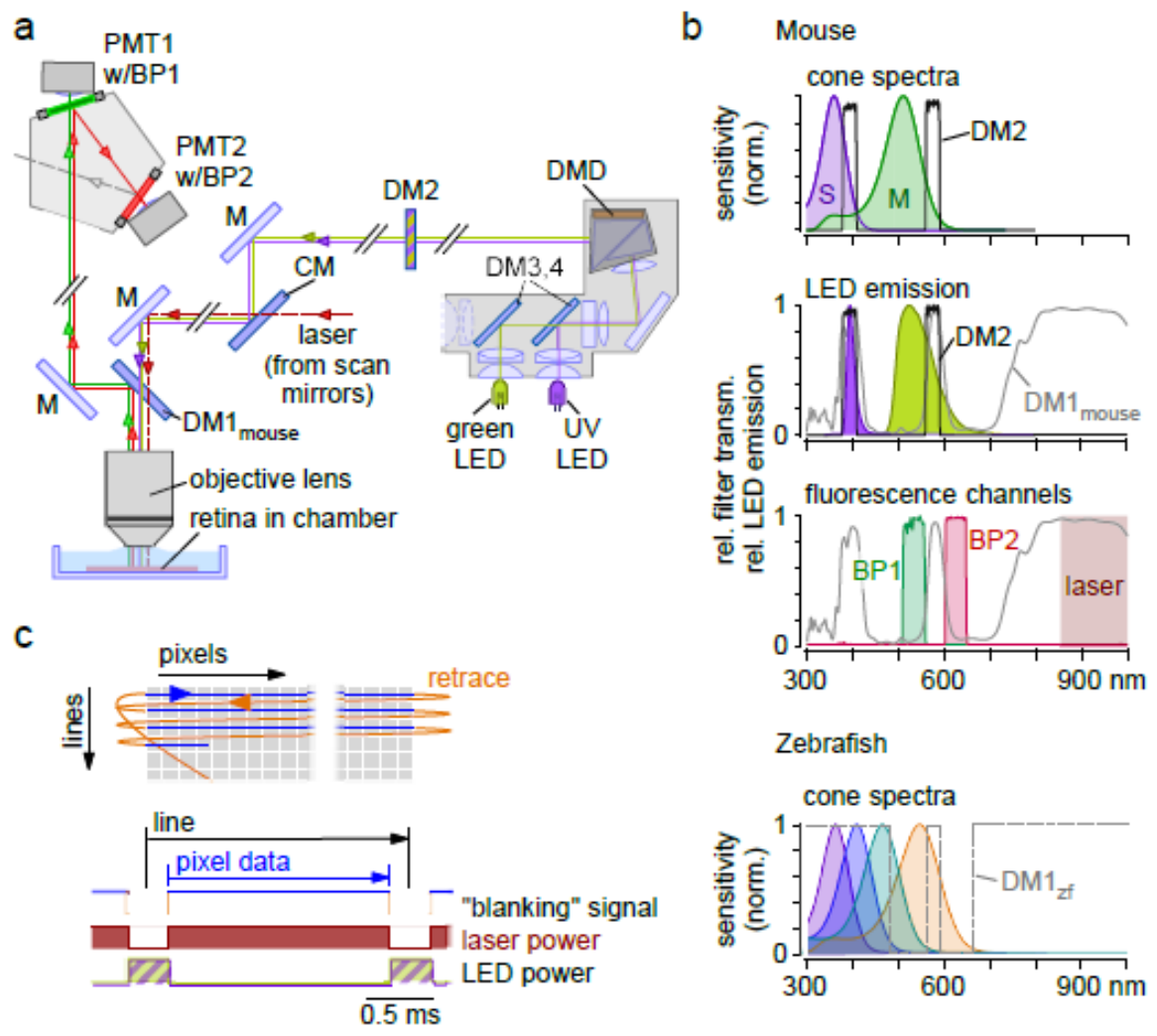

Zebrafish

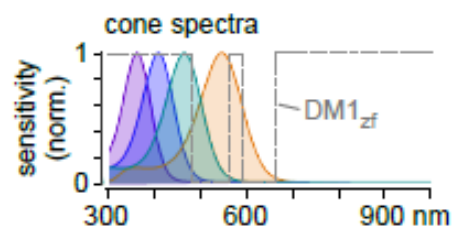

Fig. 5. Optical pathways

The combination of the band-pass filter (DM2), fluorescence emission filters (BP1, BP2) and the custom-designed dichroic mirror (DM1, as mouse-version DM1 mouse available as z400/580/890Tpc from AHF on request) directly above the objective lens takes care of spectral separation between (i) stimulus light and (ii) IR excitation laser, both of which are transmitted by DM1 in different bands, and (iii) the probe's fluorescence, which is reflected by DM1 towards the PMTs (Fig. 5b). Mice feature two spectral types of cone photoreceptor [69], expressing a short wavelength-sensitive (S-, "UV") and a medium wavelength-sensitive (M-, "green") opsin with peak sensitivities at 360 and $510 \mathrm{~nm}$ [62], respectively. Mouse rod sensitivity peaks very close to that of its M-cones. The "mouse" version $\mathrm{DM} 1_{\text {mouse }}$ features two narrow transmission bands for stimulation centred at $\sim 400$ and $580 \mathrm{~nm}$ (Fig. $5 b$, top). The transmission bands are not aligned with the peak sensitivities of the mouse opsins to allow for two broad detection bands (see broad "troughs" in DM1 $1_{\text {mouse }}$ transmission at approx. 450550 and 600-700 nm; Fig. 5b, bottom) for standard "green" and "red" fluorescent probes. While not 
ideal because higher LED intensities are needed to drive the cones, this "compromise" has been successfully used for chromatic stimulation of the mouse retina (e.g. [59, 87]). To increase stimulation efficiency of the mouse's S-cone, using shorter-wavelength LEDs (e.g. $360 \mathrm{~nm}$ ) is tempting, however, we recommend checking first if the chosen projector sufficiently modulates/transmits such short wavelengths - in the DMD projectors we tested (e.g. DLP LightCrafter 4500; K11 by Acer Computer GmbH, Ahrensburg, Germany), transmission sharply drops at $\sim 380 \mathrm{~nm}$. In any case, careful calibration of the effective photoisomerisation rates induced by the stimulator channels is mandatory, in particular when studying chromatic processing (for details and procedures, see e.g. $[59,87,88])$.

It is possible to adapt the filter/LED combinations for TTO stimulation also to the specific spectral requirements of other species. A possible filter design for zebrafish ( $D M 1_{2 f}$, Fig. $5 b$, bottom) could use the transmission band at $\sim 590 \mathrm{~nm}$ of $\mathrm{DM} 1_{\text {mouse }}$ for stimulation of the fish red opsin, whereas the 400-nm transmission of DM1 mouse would need to be broadened to allow stimulating the fish UV, blue and green opsins. As long as temporally modulated visual stimuli suffice, such a tetrachromatic stimulator can be easily designed (cf. Section 2.2). For tetrachromatic, spatio-temporally structured stimuli, two DMD projectors equipped with a complementary set of LEDs can be combined. The selection of dichroic filter combinations for restricting the sometimes quite broad LED emission spectra to narrow bands - to accommodate for the necessary fluorescence detection channels - can become here more difficult, as it may necessitate (costly) custom filter designs.

Unfortunately, spectral separation only reduces, but does not eliminate stimulus light-evoked artefacts in the PMTs (cf. Section 2.2), mainly because filters are imperfect and PMTs highly lightsensitive. Therefore, we also use temporal separation of fluorescent signal detection and visual stimulation (Fig. 5c) by enabling the LEDs only during the retrace period of the microscope's scan mirrors. This "blanking" signal is generated by the imaging software (in parallel to the signals that drive the scanners) and can also be used to decrease the laser power during retrace (e.g. with a Pockels cell), helping to minimise the exposure of the retina to the excitation laser. To avoid that the 
resulting "flicker" of the LEDs modulate the retina's activity, we use a total scan line duration of $2 \mathrm{~ms}$ (e.g. $1.6 \mathrm{~ms}$ for signal acquisition, $0.4 \mathrm{~ms}$ for the retrace/visual stimulation); the resulting blanking signal frequency of $500 \mathrm{~Hz}$ is about one order of magnitude faster than the flicker fusion frequency of most mammals [89]. The LEDs can be switched either electronically (e.g. a "breaker" circuit) or mechanically (e.g. using a "chopper" wheel, that is synchronised with the blanking signal; see MC2000B, Thorlabs GmbH, Dachau, Germany). If the LEDs' current requirements are high, building a breaker circuit with the required temporal precision becomes increasingly difficult; in this case an optical chopper solution may be considered. The latter, however, tends to be less flexible with respect to the timing and a potential source of vibrations which can be ameliorated by using rubber feet.

A focus adjustment lens in the stimulus pathway (not shown in Fig. 5a; for details, see [3]) introduces a vertical offset between focal plane (e.g. in the IPL) and stimulation plane in the photoreceptor outer segment layer (cf. Fig. 6a). In principle, it is possible to couple this adjustment lens to the movement of the objective (e.g. by using an electrically tuneable lens) to allow for changing the focal plane while keeping the stimulation plane fixed. In practice, we found that small changes of the focal plane - for example by $\sim 50 \mu \mathrm{m}$ to scan different levels of the IPL - has a negligible effect on stimulus quality.

\subsection{Estimating and ameliorating laser effects on retinal activity}

As discussed in Section 1.2, the laser used to excite the fluorescent probes can evoke substantial light responses in the retina's photoreceptors via three mechanisms: direct $1 \mathrm{P}$ and $2 \mathrm{P}$ excitation, as well as indirect excitation by the emitted fluorescence generated in the tissue. Light responses due to direct $1 \mathrm{P}$ and $2 \mathrm{P}$ excitation were first described when imaging whole-mounted salamander retina [2] and later quantified together with indirect excitation in more detail in the rabbit retinal whole-mount [3]. In the following, we discuss the extent of photoreceptor stimulation by the different mechanisms and give pointers for minimising these effects. 
Generally, direct 1P excitation of the photopigment by the laser cannot be avoided, as the opsins' spectral sensitivity curves represent probability distributions, which means the chance of excitation is non-zero even for wavelengths in the IR range. However, this probability rapidly falls with increasing laser wavelengths [90]. For example, at $930 \mathrm{~nm}$ mouse rhodopsin is in the order of $10^{-11}$ times less sensitive than at $497 \mathrm{~nm}$, the peak of its sensitivity curve. This amounts to a rate of around 20 photoisomerisations per second and $\operatorname{rod}\left(\mathrm{R}^{*} \mathrm{~s}^{-1} \operatorname{rod}^{-1}\right)$ for a $5 \mathrm{~mW}$-laser beam scanning a wholemounted retina with the focal plane in the IPL (Fig. 6a,b; for the detailed calculation, see [3]). Of course, when scanning closer to the photoreceptor layer, the laser-illuminated area in the photoreceptor layer decreases quadratically with decreasing distance, resulting in a higher photon density and, therefore, in an increased 1P-dependent photoisomerisation rate in photoreceptors within that area.

Two-photon excitation requires a high photon density, which in practice is generated by pulsing an IR laser that is, "compressing" the laser's power into very short light pulses. As 2P excitation probability falls with the fourth power with increasing distance from the focal plane, fluorophores are only excited in a tiny volume $(<1 \mathrm{fl})$ within the focal plane (2P-based optical sectioning, see $[1,91])$. For the same reason, it might seem that direct $2 \mathbf{P}$ excitation of the photoreceptor layer is unlikely. However, due to the extreme photopigment concentration in the photoreceptor outer segment (e.g. mouse rods contain in the order of $7 \cdot 10^{7}$ rhodopsin molecules [92]), combined with the high gain of the phototransduction cascade [93], even a few 2P excitation events can trigger sizable light responses, as have been observed in isolated rods [94]. A back-of-the-envelope calculation for mouse rods suggests $2 \mathrm{P}$ excitation-related photoisomerisation rates of $2 \cdot 10^{4} \mathrm{R}^{*} \mathrm{~s}^{-1} \operatorname{rod}^{-1}$ at $5 \mathrm{~mW}$ laser power [3], which is 1,000 times higher compared to the estimate for 1P excitation. Experimentally, the relative effect sizes of $1 \mathrm{P}$ vs. $2 \mathrm{P}$ photoreceptor excitation can be evaluated, for instance, by electrically recording spiking activity in RGCs in the absence of any fluorescent dye, while scanning the tissue with the excitation laser in mode-lock (pulsing) or continuous mode (see Fig. 7 in [3]). Without the laser being pulsed, $2 \mathrm{P}$ absorption events becomes so unlikely that any remaining laser 
effect on the RGC activity should result from the photoreceptors "seeing" the $930 \mathrm{~nm}$-laser (1P excitation).

In addition to the dependence on absolute laser power, area and depth of the scanned field (or, more precisely, volume) within the retinal whole-mount are important factors that determine the laser-evoked activity observed under a specific experimental condition. Generally, the smaller the distance between the scanning plane and the layer of the photoreceptor outer segments, the stronger the laser-evoked $1 \mathrm{P}$ and, in particular, 2P effects. In practice, light stimulus-evoked activity in the OPL fades within a couple of seconds when using mouse retinal whole-mounts, but activity imaging in any other layer proximal to the OPL (that is, closer to or in the $\mathrm{GCL}$ ) works quite well. The lack of discernible, stable light responses in the OPL may be due to excessive photopigment bleaching by the laser and/or that stimulus-evoked activity simply gets drowned in the laser-evoked activity. Finally, the combination of scan field depth and size determine the extent of the laserilluminated area at the level of the photoreceptor outer segments; if this area is in the same size range as the receptive fields (RFs) of the recorded neurons, comparatively small changes in scan depth and/or area may have also rather unexpected consequences for the laser-evoked activity: For example, when moving the focal plane more proximally, the laser-illuminated area at the outer segment level grows and the electrically-recorded laser-evoked response in, say, an RGC first increases until the laser-illuminated area covers the cell's RF centre, and then decreases when the laser-illuminated area enters the cell's antagonistic RF surround. Eventually, the response might even change polarity if the laser stimulation of the RF surround overwhelms the RF centre response (for an example, see Fig. 7 in [3]). 


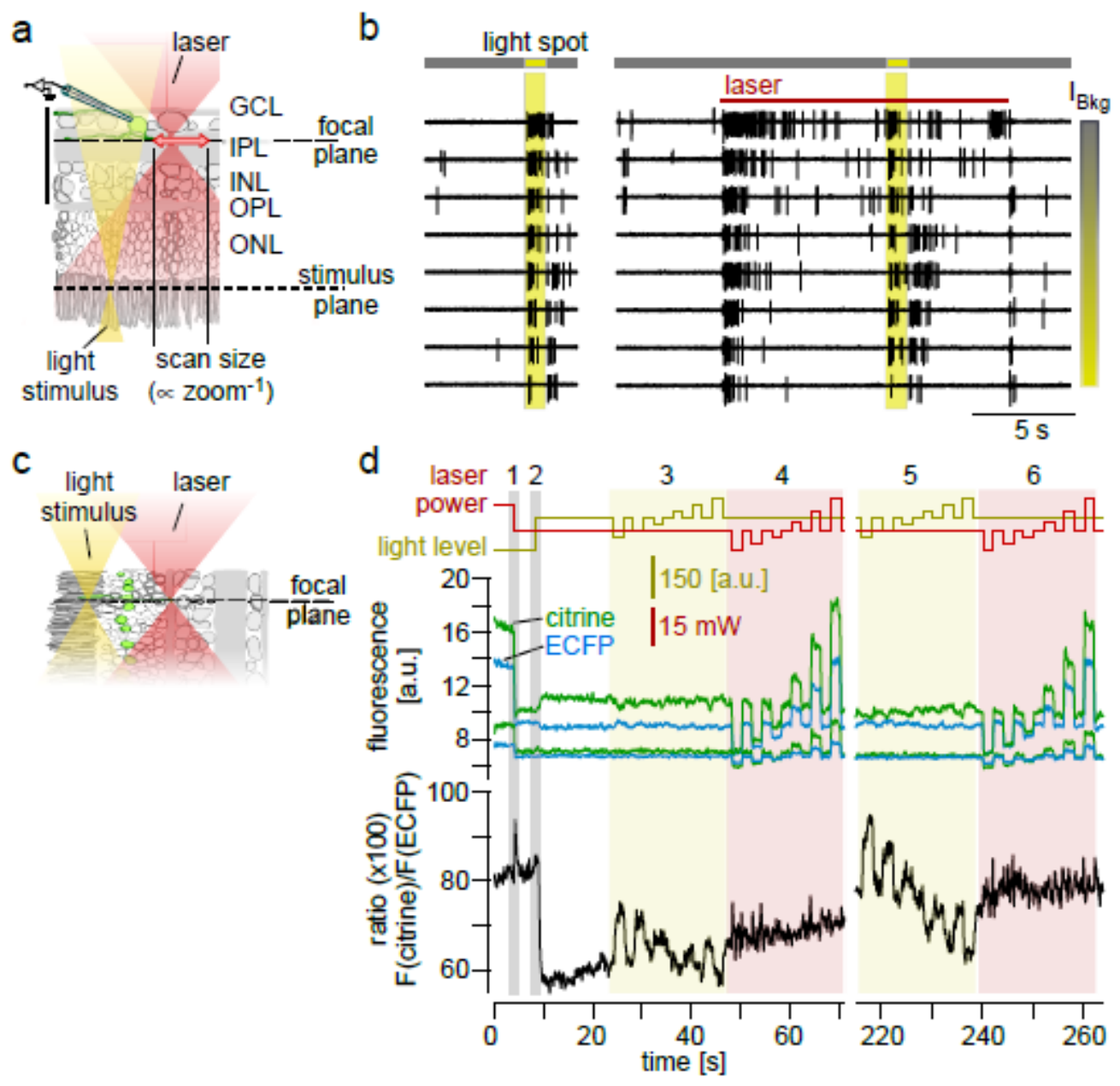

Fig. 6. 2P effects

Any fluorescence emitted within the tissue can lead to indirect excitation of the photoreceptors. This effect, however, is quite variable but tends to be in the range of that for direct 1P excitation. The amount of activity caused by indirect excitation is difficult to predict, because it depends on many factors related to the experimental situation, including dye concentration, the scanned volume from which the fluorescence is emitted, and how much of the fluorescence is scattered towards the photoreceptor layer. Also, the homogeneity of the labelling within the scanned tissue volume matters; for instance, the presence of a highly fluorescent isolated structure (e.g. a dye-filled soma) in that volume can lead to rhythmic laser-evoked activity in the retinal network caused by the periodic modulation of emitted fluorescence, in particular when using low scan rates. While most of these factors cannot be easily optimised, it may be possible to reduce the fluorescence generated to 
the minimum required for a sufficient signal-to-noise ratio $(\mathrm{S} / \mathrm{N})$ in the signal of interest by carefully adjusting the laser power and restricting the scanned volume to the necessary minimum. The success of these efforts, of course, also depends on the sensitivity and fidelity of the fluorescence detection system (for an example of how to estimate the effect of indirect excitation as a function of $S / N$, see $[3])$.

Because slicing unavoidably damages the retinal network, whole-mounts are preferable over retinal slices - at least for circuit-oriented research questions (cf. Sections 1.4 and 3.1). However, if optical recordings in the OPL are needed, vertical slices represent a viable alternative (e.g. $[4,51,59])$. By restricting the scan field to the OPL, the perpendicular orientation of the laser beam to the slice surface eliminates in-path laser illumination of the photoreceptor layer (Fig. 6c). Since only scattered laser light can reach the photopigments, direct excitation-related effects are strongly reduced compared to the whole-mount situation, enabling direct recordings of light stimulus-driven photoreceptor activity (Figs. 4a, 6d). Under the assumption that the focal volume roughly behaves like a point source (with fluorescence radiating equally likely in all directions), indirect laser effects are expected to be similar in the slice and the whole-mount.

Independent of the recording configuration of choice, we highly recommend checking carefully for laser-evoked contributions to the signal of interest. Here, it is also important to acknowledge that not all neuron types may have the same sensitivity to laser effects. Wherever feasible, the combined effect of direct and indirect excitation may best be first evaluated using electrical recordings (e.g. with and without fluorescent labelling) (cf. Fig. 6b). This also allows to check if the cells' activity returns to the pre-laser baseline after the initial laser-induced activity transient. Here, a crucial measure for reducing the impact of laser effects on the recorded activity is to provide sufficiently strong, steady background illumination via the light stimulator: By comparing the activity before and during laser scanning while titrating with different background levels, it is possible to reduce the effects to a short laser onset-evoked transient that settles to the pre-laser baseline level within seconds, while preserving the characteristics of the light responses without the laser (e.g. $3^{\text {rd }}$ row 
from the bottom in Fig. 6b). In practice, this also means that stimulus presentations should always be preceded by an adequate period of time of laser scanning and background illumination (cf. Fig. $6 \mathrm{~d}$ ). In conclusion, several measures can be taken to reduce direct $1 \mathrm{P}$ and $2 \mathrm{P}$, as well as indirect laserevoked effects:

(i) Limit the laser power to levels adequate to achieve sufficient $\mathrm{S} / \mathrm{N}$ in the signal of interest;

(ii) Present a steady background illumination using the stimulator to reduce the overall light sensitivity of the retina;

(iii) After laser onset, give the retinal network sufficient time to adapt to the combined laser/stimulator background;

(iv) Restrict scan volume size as much as possible;

(v) If feasible, avoid highly fluorescent structures and/or homogeneities in fluorescent labelling within the scan volume.

By considering these measures, laser-evoked responses can often be reduced to a transient that no longer interferes with the actual light stimulus-evoked activity. Nevertheless, due to the unavoidable presence of background illumination, and, in particular, the large contribution of $2 \mathrm{P}$ photopigment excitation to the laser effects, imaging of light-evoked retinal activity remains restricted to the mesopic/photopic range. 


\section{References}

1. Denk W., Strickler J.H., Webb W.W., et al. (1990) Two-photon laser scanning fluorescence microscopy. Science 248(4951):73-6

2. Denk W., Detwiler P.B. (1999) Optical recording of light-evoked calcium signals in the functionally intact retina. Proc Natl Acad Sci U S A 96:7035-40

3. Euler T., Hausselt S.E., Margolis D.J., et al. (2009) Eyecup scope-optical recordings of light stimulus-evoked fluorescence signals in the retina. Pflugers Arch Eur J Physiol 457:1393-1414

4. Wei T., Schubert T., Paquet-Durand F., et al. (2012) Light-driven calcium signals in mouse cone photoreceptors. J Neurosci 32:6981-6994

5. Akerboom J., Chen T.W., Wardill T.J., et al. (2012) Optimization of a GCaMP calcium indicator for neural activity imaging. J Neurosci 32:13819-13840

6. Chen T.-W., Wardill T.J., Sun Y., et al. (2013) Ultrasensitive fluorescent proteins for imaging neuronal activity. Nature 499:295-300

7. Marvin J.S., Borghuis B.G., Tian L., et al. (2013) An optimized fluorescent probe for visualizing glutamate neurotransmission. Nat Methods 10:162-170

8. Wu J., Abdelfattah A.S., Zhou H., et al. (2018) Genetically encoded glutamate indicators with altered color and topology. ACS Chem Biol. doi: 10.1021/acschembio.7b01085

9. Marvin J.S., Scholl B., Wilson D.E., et al.(2017) Stability, affinity and chromatic variants of the glutamate sensor iGluSnFR. bioRxiv. doi: 10.1101/235176

10. Dreosti E., Odermatt B., Dorostkar M.M., et al. (2009) A genetically encoded reporter of synaptic activity in vivo. Nat Methods 6:883-9

11. Baden T., Esposti F., Nikolaev A., et al. (2011) Spikes in retinal bipolar cells phase-lock to visual stimuli with millisecond precision. Curr Biol 21:1859-1869

12. Esposti F., Johnston J., Rosa J.M., et al. (2013) Olfactory stimulation selectively modulates the OFF pathway in the retina of zebrafish. Neuron 79:97-110

13. Nikolaev A., Leung K.-M., Odermatt B., et al. (2013) Synaptic mechanisms of adaptation and sensitization in the retina. Nat Neurosci 16:934-41

14. Odermatt B., Nikolaev A., Lagnado L. (2012) Encoding of luminance and contrast by linear and nonlinear synapses in the retina. Neuron 73:758-73

15. Rosa J.M., Ruehle S., Ding H., et al. (2016) Crossover inhibition generates sustained visual responses in the inner retina. Neuron 90(2):308-19

16. Zimmermann M.J., Nevala N.E., Yoshimatsu T., et al. (2017) Zebrafish differentially process colour across visual space to match natural scenes. bioRxiv. doi: 10.1101/230144

17. Antinucci P., Suleyman O., Monfries C., et al. (2016) Neural mechanisms generating orientation selectivity in the retina. Curr Biol 26:1802-1815

18. Hasan M.T., Friedrich R.W., Euler T., et al. (2004) Functional fluorescent $\mathrm{Ca}^{2+}$ indicator proteins in transgenic mice under TET control. PLoS Biol 2:e163

19. Chen Q., Pei Z., Koren D., et al. (2016) Stimulus-dependent recruitment of lateral inhibition underlies retinal direction selectivity. eLife 5:1-19

20. Yonehara K., Farrow K., Ghanem A., et al. (2013) The first stage of cardinal direction selectivity is localized to the dendrites of retinal ganglion cells. Neuron 79:1078-1085

21. Vaney D.I., Sivyer B., Taylor W.R. (2012) Direction selectivity in the retina: symmetry and 
asymmetry in structure and function. Nat Rev Neurosci 13:194-208

22. Duebel J., Haverkamp S., Schleich W., et al. (2006) Two-photon imaging reveals somatodendritic chloride gradient in retinal ON-type bipolar cells expressing the biosensor clomeleon. Neuron 49:81-94

23. Borghuis B.G., Marvin J.S., Looger L.L., et al. (2013) Two-photon imaging of nonlinear glutamate release dynamics at bipolar cell synapses in the mouse retina. J Neurosci 33:1097210985

24. Franke K., Berens P., Schubert T., et al. (2017) Inhibition decorrelates visual feature representations in the inner retina. Nature 542:439-44

25. Borghuis B.G., Looger L.L., Tomita S., et al. (2014) Kainate receptors mediate signalling in both transient and sustained OFF bipolar cell pathways in mouse retina. J Neurosci 34:6128-6139

26. Park S.J., Kim I.J., Looger L.L., et al. (2014) Excitatory synaptic inputs to mouse on-off direction-selective retinal ganglion cells lack direction tuning. J Neurosci 34:3976-3981

27. Euler T., Detwiler P.B., and Denk W. (2002) Directionally selective calcium signals in dendrites of starburst amacrine cells. Nature 418:845-852

28. Ding H., Smith R.G., Poleg-polsky A., et al. (2016) Species-specific wiring for direction selectivity in the mammalian retina. Nature 535:1-17

29. Jackman S.L., Babai N., Chambers J.J., et al. (2011) A positive feedback synapse from retinal horizontal cells to cone photoreceptors. PLoS Biol 9:e1001057

30. Baden T., Berens P., Franke K., et al. (2016) The functional diversity of retinal ganglion cells in the mouse. Nature 529:345-350

31. Briggman K.L., Euler T. (2011) Bulk electroporation and population calcium imaging in the adult mammalian retina. J Neurophysiol 105:2601-2609

32. Briggman K.L., Helmstaedter M., Denk W. (2011) Wiring specificity in the direction-selectivity circuit of the retina. Nature 471:183-188

33. Baden T., Berens P., Bethge M., et al. (2013) Spikes in mammalian bipolar cells support temporal layering of the inner retina. Curr Biol 23:48-52

34. Poleg-Polsky A., Diamond J.S. (2016) Retinal circuitry balances contrast tuning of excitation and inhibition to enable reliable computation of direction selectivity. J Neurosci 36:5861-5876

35. Kemmler R., Schultz K., Dedek K., et al. (2014) Differential regulation of cone calcium signals by different horizontal cell feedback mechanisms in the mouse retina. J Neurosci 34:11826-43

36. Chapot C.A., Behrens C., Rogerson L.E., et al. (2017) Local signals in mouse horizontal cell dendrites. Curr Biol 27:3603-3615.e5

37. Baden T., Nikolaev A., Esposti F., et al. (2014) A Synaptic mechanism for temporal filtering of visual signals. PLoS Biol 12:e1001972

38. Grimes W.N., Zhang J., Graydon C.W., et al. (2010) Retinal parallel processors: more than 100 independent microcircuits operate within a single interneuron. Neuron 65:873-885

39. Hausselt S.E., Euler T., Detwiler P.B., et al. (2007) A dendrite-autonomous mechanism for direction selectivity in retinal starburst amacrine cells. PLoS Biol 5:e185

40. Hsiang J.-C., Johnson K., Madisen L., et al. (2017) Local processing of visual information in neurites of VGluT3-expressing amacrine cells. Elife e31307

41. Lee S., Zhang Y., Chen M., et al. (2016) Segregated glycine-glutamate co-transmission from vGluT3 amacrine cells to contrast-suppressed and contrast-enhanced retinal circuits. Neuron 
90:27-34

42. Auferkorte O.N., Baden T., Kaushalya S.K., et al. (2012) GABA(A) receptors containing the alpha2 subunit are critical for direction-selective inhibition in the retina. PLoS One 7:e35109

43. Oesch N., Euler T., Taylor W.R. (2005) Direction-selective dendritic action potentials in rabbit retina. Neuron 47:739-750

44. Solovei I., Kreysing M., Lanctôt C., et al. (2009) Nuclear architecture of rod photoreceptor cells adapts to vision in mammalian evolution. Cell 137:356-368

45. Jacobs G.H. (2012) The Evolution of Vertebrate Color Vision, In: López-Larrea J (ed) Sensing in Nature. Advances in Experimental Medicine and Biology, Springer, New York, NY, vol 739, pp. 156-172

46. Lewis P.R. (1955) A theoretical interpretation of spectral sensitivity curves at long wavelengths. J Physiol 130:45-52

47. Göppert-Mayer M. (1931) Über Elementarakte mit zwei Quantensprüngen. Ann Phys 9:273295

48. Reperant J., Ward R., Miceli D., et al. (2006) The centrifugal visual system of vertebrates: a comparative analysis of its functional anatomical organization. Brain Res Rev 52:1-57

49. Werblin F.S. (1978) Transmission along and between rods in the tiger salamander retina. J Physiol 280:449-470

50. Kulkarni M., Schubert T., Baden T., et al. (2015) Imaging Ca ${ }^{2+}$ dynamics in cone photoreceptor axon terminals of the mouse retina. J Vis Exp e52588

51. Kulkarni M., Trifunović D., Schubert T., et al. (2016) Calcium dynamics change in degenerating cone photoreceptors. Hum Mol Genet 25:3729-3740

52. Lin B., Masland R.H. (2006) Populations of wide-field amacrine cells in the mouse retina. J Comp Neurol 499:797-809

53. Peichl L., González-Soriano J. (1994) Morphological types of horizontal cell in rodent retinae: a comparison of rat, mouse, gerbil and guinea pig. Vis Neurosci 11:501-517

54. Yin L., Masella B., Dalkara D., et al. (2014) Imaging light responses of foveal ganglion cells in the living macaque eye. J Neurosci 34:6596-6605

55. Palczewska G., Dong Z., Golczak M., et al. (2014) Non-invasive two-photon microscopy imaging of mouse retina and retinal pigment epithelium through the pupil of the eye. Nat Med 20:785-789

56. Antinucci P., Hindges R. (2016) A crystal-clear zebrafish for in vivo imaging. Sci Rep 6:29490

57. Brainard D.H. (1997) The Psychophysics Toolbox. Spat Vis 10:433-6

58. Breuninger T., Puller C., Haverkamp S., et al. (2011) Chromatic bipolar cell pathways in the mouse retina. J Neurosci 31:6504-6517

59. Baden T., Schubert T., Chang L., et al. (2013) A tale of two retinal domains: Near-optimal sampling of achromatic contrasts in natural scenes through asymmetric photoreceptor distribution. Neuron 80:1206-1217

60. Ivanova E., Toychiev A.H., Yee C.W., et al. (2013) Optimized protocol for retinal wholemount preparation for imaging and immunohistochemistry. J Vis Exp e51018

61. Arey L.B. (1916) The movements in the visual cells and retinal pigment of the lower vertebrates. J Comp Neurol 26:121-201

62. Jacobs G.H., Neitz J., Deegan J.F. (1991) Retinal receptors in rodents maximally sensitive to 
ultraviolet light. Nature 353:655-656

63. Wang J.-S., Kefalov V.J. (2011) The cone-specific visual cycle. Prog Retin Eye Res 30:115-128

64. Li P.H., Field G.D., Greschner M., et al. (2014) Retinal representation of the elementary visual signal. Neuron 81:130-9

65. Newman E.A., Bartosch R. (1999) An eyecup preparation for the rat and mouse. J Neurosci Methods 93:169-75

66. Boycott B.B., Wässle H. (1974) The morphological types of ganglion cells of the domestic cat's retina. J Physiol 240:397-419

67. Goodchild A.K., Ghosh K.K., Martin P.R. (1996) Comparison of photoreceptor spatial density and ganglion cell morphology in the retina of human, macaque monkey, cat, and the marmoset Callithrix jacchus. J Comp Neurol 366:55-75

68. Peichl L. (2005) Diversity of mammalian photoreceptor properties: Adaptations to habitat and lifestyle? Anat Rec - Part A Discov Mol Cell Evol Biol 287:1001-1012

69. Szél A., Röhlich P., Caffé A.R.R., et al. (1992) Unique topographic separation of two spectral classes of cones in the mouse retina. J Comp Neurol 325:327-342

70. Bleckert A., Schwartz G.W., Turner M.H., et al. (2014) Visual space is represented by nonmatching topographies of distinct mouse retinal ganglion cell types. Curr Biol 24:310-315

71. Rousso D.L., Qiao M., Kagan R.D., et al. (2016) Two pairs of ON and OFF retinal ganglion cells are defined by intersectional patterns of transcription factor expression. Cell Rep 15:19301944

72. Zhang Y., Kim I.-J., Sanes J.R., et al. (2012) The most numerous ganglion cell type of the mouse retina is a selective feature detector. Proc Natl Acad Sci 109:E2391-E2398

73. Joesch M., Meister M. (2016) A neuronal circuit for colour vision based on rod-cone opponency. Nature 532:236-239

74. Wei W., Elstrott J., Feller M.B. (2010) Two-photon targeted recording of GFP-expressing neurons for light responses and live-cell imaging in the mouse retina. Nat Protoc 5:1347-1352

75. Sterratt D.C., Lyngholm D., Willshaw D.J., et al. (2013) Standard anatomical and visual space for the mouse retina: Computational reconstruction and transformation of flattened retinae with the Retistruct package. PLoS Comput Biol 9

76. Sabbah S., Gemmer J.A., Bhatia-Lin A., et al. (2017) A retinal code for motion along the gravitational and body axes. Nature 546:492-497

77. Schlichtenbrede F.C., Mittmann W., Rensch F., et al. (2009) Toxicity assessment of intravitreal triamcinolone and bevacizumab in a retinal explant mouse model using two-photon microscopy. Invest Ophthalmol Vis Sci 50:5880-7

78. Lin M.Z., Schnitzer M.J. (2016) Genetically encoded indicators of neuronal activity. Nat Neurosci 19:1142-53

79. Bethge P., Carta S., Lorenzo D.A., et al. (2017) An R-CaMP1.07 reporter mouse for cell-typespecific expression of a sensitive red fluorescent calcium indicator. PLoS One 12:e0179460

80. Davidson B.L., Breakefield X.O. (2003) Neurological diseases: Viral vectors for gene delivery to the nervous system. Nat Rev Neurosci 4:353-364

81. Vandenberghe L.H., Auricchio A. (2012) Novel adeno-associated viral vectors for retinal gene therapy. Gene Ther 19:162-8

82. Zhao Y., Araki S., Wu J., et al. (2011) An expanded palette of genetically encoded $\mathrm{Ca}^{2+}$ 
indicators. Science 96333:1888-91

83. Dalkara D., Byrne L.C., Klimczak R.R., et al. (2013) In vivo-directed evolution of a new adenoassociated virus for therapeutic outer retinal gene delivery from the vitreous. Sci Transl Med 5:189ra76-189ra76

84. Lavis L.D. (2017) Teaching old dyes new tricks: Biological probes built from fluoresceins and rhodamines. Annu Rev Biochem 86:825-843

85. Tada M., Takeuchi A., Hashizume M., et al. (2014) A highly sensitive fluorescent indicator dye for calcium imaging of neural activity in vitro and in vivo. Eur J Neurosci 39:1720-1728

86. Chang L., Breuninger T., Euler T. (2013) Chromatic coding from cone-type unselective circuits in the mouse retina. Neuron 77:559-571

87. Wang Y. V, Weick M., Demb J.B. (2011) Spectral and temporal sensitivity of cone-mediated responses in mouse retinal ganglion cells. J Neurosci 31:7670-7681

88. Healy K., McNally L., Ruxton G.D., et al. (2013) Metabolic rate and body size are linked with perception of temporal information. Anim Behav 86:685-696

89. Lamb T.D. (1995) Photoreceptor spectral sensitivities: Common shape in the long-wavelength region. Vision Res 35:3083-3091

90. Denk W., Svoboda K. (1997) Photon upmanship: Why multiphoton imaging is more than a gimmick. Neuron 18:351-357

91. Lyubarsky A.L., Daniele L.L., Pugh Jr. E.N. (2004) From candelas to photoisomerizations in the mouse eye by rhodopsin bleaching in situ and the light-rearing dependence of the major components of the mouse ERG. Vis Res 44:3235-3251

92. Luo D.G., Xue T., Yau K.W. (2008) How vision begins: An odyssey. Proc Natl Acad Sci U S A 105:9855-9862

93. Gray-Keller M., Denk W., Shraiman B., et al. (1999) Longitudinal spread of second messenger signals in isolated rod outer segments of lizards. J Physiol 519 Pt 3:679-692

94. Mank M., Reiff D.F., Heim N., et al. (2006) A FRET-based calcium biosensor with fast signal kinetics and high fluorescence change. Biophys J 90:1790-1796

95. Zariwala H.A., Borghuis B.G., Hoogland T.M., et al. (2012) A Cre-dependent GCaMP3 reporter mouse for neuronal imaging in vivo. J Neurosci 32:3131-3141 


\section{Figure Legends}

Fig. 1 Retina and retinal labelling. (a) Schematic cross-section of a mammalian retina (c, cone photoreceptor; $r$, rod photoreceptor; $h$, horizontal cell; $b$, bipolar cell; $a$, amacrine cell; $g$, retinal ganglion cell; OS+IS, outer and inner segments; ONL, outer nuclear layer; OPL, outer plexiform layer; INL, inner nuclear layer; IPL, inner plexiform layer; GCL, ganglion cell layer; NFL, nerve fibre layer). The following panels illustrate methods for labelling retinal neurons (for details, see text): (b) Top view of rabbit starburst amacrine cell (SAC) injected with the synthetic $\mathrm{Ca}^{2+}$ indicator OGB-1. (c) Whole-mount retina of transgenic mouse expressing tdTomato (red) in starburst amacrine cells (SACs), and ubiquitously expressing the genetically encoded $\mathrm{Ca}^{2+}$ biosensor GCaMP6f (green) introduced by viral infection. The tissue was counterstained with sulforhodamine (SR101, red), here visualizing the blood vessels (cf. Fig. 3). (d) Scan of the axon terminal system of a bipolar cell filled with Alexa 555 (red) via a sharp electrode in the flat-mounted mouse retina, with the iGluSnFR staining overlaid in green. (e) Retinal slice from HR2.1:TN-XL mouse line with cones expressing the $\mathrm{Ca}^{2+}$ biosensor TN-XL. (f) iGluSnFR sparsely expressed under the RibeyeA promotor labels both photoreceptors and bipolar cells in larval zebrafish in vivo (7 days post fertilisation, courtesy of T. Yoshimatsu, Univ. Sussex, Brighton, UK). $\left(\mathbf{g}_{\mathbf{1}, \mathbf{2}}\right)$ Mouse retinal whole-mount electroporated with OGB1 (green) and counterstained with SR101 (red), here visualizing the vessels. (h) Retina from a same mouse line as in (c), with SACs expressing tdTomato (red) and bipolar cells sparsely loaded with OGB1 (green) via dye incubation (courtesy of J. Diamond, NINDS, Bethesda, MA, USA; for protocol, see [34]). Scale bars: b, c, $g_{2}, h, 50 \mu \mathrm{m} ; \mathrm{d}, \mathrm{e}, 10 \mu \mathrm{m} ; \mathrm{g}_{1}, 300 \mu \mathrm{m} ; \mathrm{f}, 20 \mu \mathrm{m}$. Panel (d) adapted from Franke et al. 2017 [24] with permission from Springer Nature. Panel (e) adapted from Baden et al. 2013 [59] with permission from Elsevier. Panel (g) adapted from Baden et al. 2016 [30] with permission from Springer Nature

Fig. 2 Direct and indirect excitation of photoreceptors. Retinal cross-sections with schematic trajectories of exemplary single photons of infrared (IR) excitation laser (red) and fluorescence 
emitted from activity probe (green). Photoreceptors can get activated directly by the IR laser via single-photon (a) and two-photon events (b), as well as indirectly by emitted fluorescence in the visible range (c). Arrows indicate photon propagation direction, stars symbolise photoisomerisation events in the highlighted photoreceptor. Dotted line, focal plane of the scan

Fig. 3 Visualizing retinal structure. (a) Images at different depths of whole-mounted wildtype mouse retina recorded using a $2 \mathrm{P}$ microscope. The living, light-sensitive tissue was stained with SR101 to visualise retinal structure and blood vessels. Layers, depths and examples for retinal cells are indicated ( $a$, amacrine cell; $g$, retinal ganglion cell; $b$, bipolar cell; arrows: $1,4,7$, blood vessels; 2 , ganglion cell axon bundles; 3 , rod bipolar cell terminal; 5, rod bipolar cell soma; 6 , cone pedicle; 8 , rod spherule; NFL, nerve fibre layer; GCL, ganglion cell layer; IPL inner plexiform layer; INL, inner nuclear layer; OPL, outer plexiform layer; ONL, outer nuclear layer; OS+IS, outer and inner segments). (b) Vertical projection of stack from a ChAT:Cre $\times$ Ai $9^{\text {tdTomato }}$ mouse counterstained with SR101 illustrating the position of the choline acetyltransferase (ChAT) immunopositive bands (white) relative to blood vessels (red). Dashed lines mark IPL borders. (c) Average depth profiles of blood vessels (red) and ChAT bands (black; \pm 1 s.d. shading). Scale bars: a, $20 \mu \mathrm{m} ; \mathrm{b}, 10 \mu \mathrm{m}$. Panels (b) and (c) modified from Franke et al. 2017 [24] with permission from Springer Nature

Fig. 4 Example responses. Exemplary global (full-field) chirp stimulus-evoked light responses recorded with different activity probes in retinal neurons in mouse ( 3 to 5 individual trials overlaid with mean trace in darker colours, respectively). (a) $\mathrm{Ca}^{2+}$ (top) and glutamate signal (bottom) measured in/at a cone photoreceptor axon terminal using the ratiometric $\mathrm{Ca}^{2+}$ biosensor TN-XL ([4, 95]; HR2.1:TNXL mouse line) and the glutamate sensor iGluSnFR ([7]; ubiquitous AAV-mediated expression), respectively. (b) $\mathrm{Ca}^{2+}$ signal in a horizontal cell dendrite recorded with the $\mathrm{Ca}^{2+}$ biosensor GCaMP3 ([96]; transgenic Cx57 $7^{+/ c r e} \times$ Ai38 mouse line). (c) $\mathrm{Ca}^{2+}$ (top) and glutamate (bottom) 
responses in/at axon terminals of different On cone bipolar cell types measured with the $\mathrm{Ca}^{2+}$ biosensor GCaMP6f ([6]; floxed AAV construct in PcP2 ${ }^{\text {Cre }}$ mouse line) and iGluSnFR (ubiquitous AAVmediated expression), respectively. (d) Somatic $\mathrm{Ca}^{2+}$ response of a starburst amacrine cell measured using OGB-1 introduced by bulk electroporation [31]. (e) Dendritic $\mathrm{Ca}^{2+}$ signals of an RGC injected with OGB-1 using a sharp electrode $\left(1^{\text {st }}\right.$ row) and somatic $\mathrm{Ca}^{2+}$ responses recorded in $\mathrm{RGCs}$ using GCaMP6f (ubiquitous AAV-mediated expression, $2^{\text {nd }}$ row), and OGB-1 (bulk electroporation; $3^{\text {rd }}$ row). Panels (a; top), (b), and (e; top) courtesy of S. Pop, Y. Ran, and C. Chapot, respectively (all: Univ. Tübingen, Germany)

Fig. 5 Optical pathways. (a) Overview of optical pathways in a 2P microscope equipped with a light stimulator designed for chromatic stimulation of mouse retina. For simplicity, most lenses and some silver mirrors (M) were omitted (for a complete description, see [3]). Excitation/detection pathway: The laser (dashed red line) passes a cold mirror (CM), dichroic mirror 1 (DM1, here the mouse version, $\left.\mathrm{DM} 1_{\text {mouse }}\right)$, and the objective lens, before exciting the probe in the recording chamber. Fluorescence emitted from the probe (red/green lines) is reflected by DM1 into the detection head containing two GaAsP photomultiplier tubes (PMT), each equipped with a band pass (BP) filter. Stimulus pathway: Light from a green and a UV LED are combined (DM3,4), illuminating a digital mirror device (DMD). The grey area represents the "light engine" of a digital light processing (DLP) device (e.g. DLP LightCrafter 4500, Texas Instruments, Dallas, TX, USA), adapted for green/UV projection (by EKB Technologies Ltd, Israel). The resulting light stimulus is filtered by a dual-band filter (DM2) and coupled via CM into the laser path. (b) Spectral separation of stimulation and excitation/fluorescence detection: First row: sensitivity curves of the mouse's short- (S) and medium- (M) wavelength sensitive opsins with stimulus filter DM2. Second row: Stimulus LED emission spectra with DM1 mouse and DM2. Third row: Fluorescence detection bands and range of excitation laser. Bottom row: Possible filter design for zebrafish $\left(D M 1_{2 f}\right)$. (c) Temporal separation of stimulation and detection, illustrated for simple frame scans (top; grey squares represent pixels): Each scan line is 
divided into two sections: one for acquiring pixel data (blue) and one for allowing the scan mirrors to move to the start of the next line ("retrace"; orange). Bottom: Signal generated by the scan software ("blanking"; top) to synchronise laser and stimulus LEDs: During retrace, laser power is blanked (centre; e.g. by a Pockels' cell), while LEDs are turned on (bottom). Schematic in (a) adapted from Euler et al. 2008 [3] with permission from Springer Nature

Fig. 6 Two-photon effects. (a) Schematic cross-section of a whole-mount retina, illustrating scan size and focal planes of light stimulus (yellow) and scanning excitation laser (red). (b) Electrophysiological cell-attached recording of a rabbit ON-OFF ganglion cell, with traces showing spiking response to a $200-\mu \mathrm{m}$ light spot alone (left), and during laser scanning (right; $\lambda=927 \mathrm{~nm}$ ) for increasing background intensities $\left(I_{\text {Bkg }}\right)$. Note the cell's responses to both laser scanning start and end. (c) Schematic cross-section of an imaging in a retinal slice. (d) $\mathrm{Ca}^{2+}$ signals recorded in axon terminals of mouse cone photoreceptors (slice preparation) exclusively expressing the ratiometric $\mathrm{Ca}^{2+}$ biosensor TN-XL $[4,95]$ (all traces represent averages of $\mathrm{n}=8$ cells). Top: Traces of light stimulus (yellow) and scanning laser power (red; $\lambda=860 \mathrm{~nm}$ ). Centre: Fluorescence signals of the two TN-XL fluorophores, citrine (green) and ECFP (cyan); lower trace pair represents background fluorescence measured outside the photoreceptors. Bottom: ratiometric signal (black), as reported by TN-XL, representing the $\mathrm{Ca}^{2+}$ level in the terminals. Reducing the laser power (1) elicited a transient in the ratio signal. Turning on the light stimulator (2) evoked a strong decrease in $\mathrm{Ca}^{2+}$ (=light response). Cones responded strongly to bright and dark flashes (3) but barely to "flashes" in scanning laser power. Laser "flashes" are strongly reflected in citrine and ECFP fluorescence, but hardly in the ratio signal. Second trial $(5,6)$ after cones had adapted to combined background caused by laser scanning and stimulator. (GCL, ganglion cell layer; IPL, inner plexiform layer; INL, inner nuclear layer; OPL, outer plexiform layer; ONL, outer nuclear layer; F, fluorescence; ECFP, enhanced cyan fluorescent protein). Panel (b) adapted from Euler et al. 2008 [3] with permission from Springer Nature; for details, see there. Panel (d) adapted from Baden et al. 2013 [59] with permission from Elsevier; for additional details, see there 